\title{
SUPERVOXEL-BASED MULTI-SCALE POINT CLOUD SEGMENTATION USING FNEA FOR OBJECT-ORIENTED ROCK SLOPE CLASSIFICATION USING TLS
}

\author{
I. Farmakis ${ }^{1 *}$, D. Bonneau ${ }^{1}$, D. J. Hutchinson ${ }^{1}$, N. Vlachopoulos ${ }^{2}$ \\ ${ }^{1}$ Dept. of Geological Sciences and Geological Engineering, Queen's University, K7K 3N6, Kingston, Ontario, Canada - \\ (farmakisig@gmail.com, david.bonneau@queensu.ca, hutchinj@queensu.ca) \\ ${ }^{2}$ Dept. of Civil Engineering, Royal Military College of Canada, K7K 7B4, Kingston, Ontario, Canada - (vlachopoulos-n@ rmc.ca)
}

Commission II, WG II/10

KEY WORDS: 3D Point Cloud, TLS, Supervoxels, Segmentation, Multi-scale, FNEA, Rock slopes

\begin{abstract}
:
Computer vision applications have been increasingly gaining space in the field of remote sensing and geosciences for automated terrain classification and semantic labelling purposes. The continuous and rapid development of monitoring techniques and enhancements in the spatial resolution of sensors have increased the demand for new remote sensing data analysis approaches. For semantic labelling of 2D (or 2.5D) image terrain representations for rock slopes, it has been shown that Object-Based Image Analysis (OBIA) results in high efficiency and accurate identification of landslide hazards. However, the application of such object-based approaches in 3D point cloud analysis is still under development for geospatial data analysis. In the field of engineering geology, which deals with complex rural landscapes, frequently the analysis needs to be conducted based solely on 3D geometrical information accounting for multiple scales simultaneously. In this study, the primary segmentation step of the object-based model is applied to a TLS-derived point cloud collected at a landslide-active rock slope. The 3D point cloud segmentation methodology proposed here builds on the principles of the Fractal Net Evolution Approach (FNEA). The objective is to provide a geometry-based point cloud segmentation framework that preserves the $3 \mathrm{D}$ character of the data throughout the process and favours the multi-scale analysis. The segmentation is performed on the basis of supervoxels based on purely geometrical local descriptors derived directly from the TLS point clouds and comprises the basis for the subsequent steps towards the development of an efficient Object-Based Point cloud Analysis (OBPA) framework in rock slope stability assessment by adding semantic meaning to the data through a homogenization process.
\end{abstract}

\section{INTRODUCTION}

Object-oriented classification approaches have become increasingly popular in the field of remote sensing and geosciences since the early 2000 s, in parallel with the growth in GIScience (Blaschke, 2010). The continuous and rapid developments in instrumentation specifications and enhancements in the spatial resolution of sensors have increased the demand for new approaches in remote sensing data analysis for terrain classification and semantic labelling of dense datasets. Object-based image analysis (OBIA - or GEOBIA for geospatial object-based image analysis) addresses problems related to traditional pixel-based image analyses of high spatial resolution image data, by initially defining objects (or segments) to classify rather than pixels (Blaschke and Tomljenović, 2012). Objects can be classified with sets of rules to define real-world objects of interest, providing higher quality and accuracy classification results. Although image segmentation is routinely used in image processing, it has gained new momentum when incorporated in object-based analysis facilitating the calculation of additional segment properties that are used as attributes for discriminating features. This primary segmentation step is usually done for one specific object scale (or model), but OBIA accommodates multiscale data handling (Blaschke, 2010). OBIA is not limited to the use of spectral information but also allows for fusion with geometric data such as derivatives from digital elevation models (DEMs), based on aerial LiDAR point clouds or UAV photogrammetry models, providing a $2.5 \mathrm{D}$ representation of the landscape.

\footnotetext{
* Corresponding author
}

In the field of engineering geology and more specifically, in landslide hazard investigation, such an additional piece of topographical and geometrical information is essential for the analysis of complex landslide-active landscapes. The 2.5D OBIA workflow has been demonstrated to be an effective and efficient method for landslide hazard identification and characterization of rotational failure mechanisms (Feizizadeh and Blaschke, 2013; Karantanellis et al., 2019). This approach has the potential to enhance landslide risk management frameworks providing a reproducible and automated process to generate a landslide inventory. Nevertheless, the investigation and categorization of other landslide hazards (i.e. rockfall) and rock slope structures such as spires and/or overhanging blocks may require fully $3 \mathrm{D}$ representation of the landscape. Downward looking 2.5D representations are not capable of capturing these features of the landscape. To capture and monitor these features on steep terrain, terrestrial laser scanners (TLS), which acquire high resolution 3D point clouds, have been used in landslide investigation in recent years (Jaboyedoff et al., 2012; Lato et al. 2012; Abellán et al., 2014; Lato et al. 2015; Telling et al., 2017).

Given the advances in remote sensing based slope monitoring for landslide hazard evaluation, a new opportunity is emerging to develop an Object-Based Pointcloud Analysis (OBPA) method for engineering geology applications. There are three main considerations for this work: i) unlike rasters, dense point clouds are inherently unstructured datasets, ii) manipulating unstructured data with millions of points is a computationally demanding task, and iii) in engineering geological applications point cloud analysis has been largely based on geometrical information previously. Point cloud analyses typically aim to 
exploit geometric signatures using low-level descriptors at userdefined neighbourhoods to label complex natural scenes using point-based machine learning classification approaches. Methods such as CANUPO (Brodu and Lague, 2012), that examines the dimensionality of each point's successively-increasing-sized neighborhood and selects the most discriminating of those, have been successfully applied to complex rock slope scenes for the classification of debris flow hazard elements (Bonneau \& Hutchinson, 2019).

The aim of the proposed research is to develop a point cloud segmentation framework for object-oriented classification specifically applied to rock slope assessments. This work utilizes supervoxels, populated with 3D geometric information derived directly from the TLS point cloud, for adding structure to the dataset, minimizing the computational time, and preserving the 3D character of the data throughout the process. The algorithm provides the user with the ability to investigate the geometric signature of the structures at multiple scales simultaneously, by means of the FNEA (Fractal Net Evolution Approach) (Baatz \& Schape, 1999), and to benefit from the semantic information becoming available.

\section{OBJECT-BASED MODEL AND LIDAR}

Object-based classification techniques have been introduced in the geoscience field as a solution able to provide higher accuracy classifications compared to pixel-based image classification techniques (Ye et al., 2018). OBIA is an image analysis technique that endeavours to mimic the human interpretation processes in identifying objects within remote sensing imagery (Blaschke et al., 2014). In many applications, important semantic information needed to interpret and understand the content of an image, is "hidden" in meaningful image objects rather than in single units of any image data structure (i.e. pixels, voxels, triangles, etc.). Thus, the task of segmenting the data into "homogenous" areas is the key component for the successful implementation of the object-based model. The objective of the segmentation process within the object-based model is to generate object primitives for classification. These objects can be considered as "new raw data" that represent either the entire real-world objects of interest or parts of them. Many algorithms and software have been developed for this purpose within the 2D domain: a review of available segmentation algorithms and software/tools for OBIA are provided by Hossain and Chen (2019).

Blaschke (2010) reports a significant increase in the publication frequency of OBIA-related studies since around 2002. Besides the availability of high-resolution remote sensing imagery, another major influence on this progress was the development and release of the commercial software package, eCognition in 2000 , which was used in about $55 \%$ of the OBIA-related scientific literature published by the year 2010. The multiresolution image segmentation algorithm (MRS) is used in the vast majority of the abovementioned applications. The MRS algorithm comprises a bottom-up region merging algorithm that accounts for the spectral homogeneity of the objects (every pixel is considered an individual object initially) as they grow at multiple scales through the Fractal Net Evolution Approach (FNEA). Moreover, the algorithm considers the object shape as another factor of the overall object homogeneity function, to a user-defined degree (see Baatz and Schape, 2000 for details).

One of the earliest ideas of applying the object-based models for LiDAR point cloud analysis exploiting the fully 3D terrain representation was implemented by Sithole and Vosselman (2003) in an urban landscape application. Their objective was to detect structures that are extensions to the bare-earth, such as bridges and trees, using primary segmentation, followed by a classification procedure. The ALS-derived data was segmented by profiling the terrain along two perpendicular directions and connecting adjacent points along the profiles by height difference and slope.

Rutzinger et al. (2008) developed an Object-Based Point cloud Analysis (OBPA) approach for urban vegetation classification, utilizing full-waveform ALS data. A seeded region merging algorithm was used for the segmentation task in that study. The neighborhood of each seed point is assumed to be $k$-sized and the merging criterion is an indirect proportional measure of echo width between neighbouring points. The growth of a segment is limited by both a user-defined maximum distance and a maximum number of points per object threshold.

More recently, Mayr et al. (2017) published the application of an OBPA framework in the field of engineering geology. Mayr et al. investigated rotational landslide hazards through object-based erosion monitoring, utilizing exclusively geometrical information derived from TLS point clouds. The segmentation is performed in two stages. The process is initiated with a primary over-segmentation by means of $k$-means clustering, with an empirical number of $k=10$, followed by a seeded region growing algorithm with randomly selected seed points. The feature space utilized in this study consisted of three low-level descriptors: $2 D / 3 D$ density ratio, omnivariance and geometric curvature. The point features are calculated within a $20 \mathrm{~cm}$ radius using point clouds of $3 \mathrm{~cm}$ average point spacing. In addition to the feature space, a 2-dimensional ( $\mathrm{x}, \mathrm{y}$ coordinates) shape threshold of \pm 0.6 $\mathrm{m}$ maximum distance to the seed point is applied to the region growing in order to keep the segments small.

In the aforementioned work on establishing an OBPA framework, it is observed that additional restrictions are applied to the segment sizes and they are not allowed to grow based on their feature space only. These restrictions are applied either as distance thresholds, pre-defined number of clusters or maximum number of points. The landslide-active slope application by Mayr et al. (2017) shows the potential of low-level local descriptors based solely on the XYZ information of the point cloud to be considered in an object-based analysis of natural terrains. In addition, other methods such as CANUPO (Brodu and Lague, 2012) have also shown that pure 3D geometrical information might permit effective natural terrain classification including classes such as rock, talus and vegetation. However, further classification of structures within the main classes may be facilitated by the use of additional object features which are generated within object-oriented classification methodologies. The objective of the work presented in this paper is to develop an efficient OBPA segmentation framework for rock slope evaluation, taking advantage of the high-resolution 3D geometrical information of TLS point clouds for subsequent object-based classification of the resulting elements. The goal of the current study is to provide a point cloud segmentation framework that preserves both the fundamental automation, reproducibility and semantic principles of the object-based model and the $3 \mathrm{D}$ character of the data throughout the process.

\section{GENERAL CONCEPT}

Segmentation is the key component of OBIA (or GEOBIA) which reduces the image complexity and produces meaningful image objects by homogenizing the content of the scene. In this study, the Fractal Net Evolution Approach is used for the segmentation task. FNEA is a widely used multi-scale segmentation algorithm that was first introduced by Baatz and Schape (1999) and very quickly became the most popular 2D image segmentation pipeline within the field of OBIA. Indicative of the popularity of the algorithm is the fact that in many cases the segmentation results of other newly proposed algorithms are 
compared with segments generated using FNEA (Hossain and Chen, 2019).

Most of the TLS-derived 3D point clouds for rock slope assessment only contain geometrical information by means of $\mathrm{XYZ}$ coordinates. Thus, in the current study, the general concept is to apply an adjustment of the FNEA in the 3D domain using exclusively geometric information instead of spectral. In that way, one can explore the evolution of the local morphometric signature over multiple scales simultaneously and take advantage of additional higher-level descriptors that can assist with the subsequent classification within the object-based model. Moreover, the resulting object sizes are freely adaptable to fit the scale of task based solely on their geometric signature within the scene and no additional size threshold is applied. Additional information such as colour, intensity or full-waveform features can be added to the analysis, but that is not within the scope of the work reported here.

\subsection{Supervoxels for 3D}

Processing of TLS point clouds, especially for natural environments is highly complex and utilizes substantial computing resources. Assigning a label to each point is a challenging exercise due to the inherently unstructured nature of the dataset. Thus, a step to provide the data with a structure is required. For this reason, the use of an octree is considered essential. Voxels, the resulting elements of the octree, are the 3D equivalent of pixels (volumetric pixel). Thus, the voxel size, which represents the resolution of the 3D geometrical information, needs to be selected according to the scale of the objects of interest. The voxel size should be selected to ensure that sufficient points are stored within each voxel to derive the geometrical information. Consequently, the 3D geometrical information resolution is directly related to the point cloud resolution (e.g. point density) which has to be adjusted to the specific needs of each application and the required level of detail. This step will markedly simplify the complexity involved in subsequent steps of point cloud segmentation and classification within the object-based model and also reduces the computational cost. Similar to the superpixel methods in computer vision, recent developments in the 3D domain compute supervoxels from a set of voxels (Aijazi et al., 2013; Li, 2018; Papon et al., 2013) (Figure 1). In the current study, supervoxels were generated using a 3D modification of the Fractal Net Evolution Approach (FNEA) which was originally developed for coloured 2D image processing. In this study geometric information is used exclusively and the spatial relationship among voxels is established based on 6 neighbourhood connectivities (e.g. common facets).

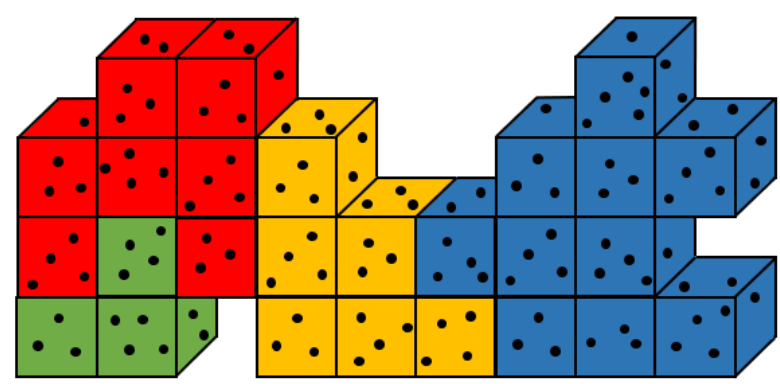

Figure 1. Representation of voxels (different cubes) and supervoxels (different colours) containing the original point cloud (dots)

\subsection{Fractal Net Evolution Approach (FNEA)}

The basic idea of the FNEA is a bottom-up region merging algorithm that aims to construct a hierarchical network of image objects (Figure 2). This process leads to the automatic extraction of object primitives at multiple levels and the knowledge-based collection of image objects on the basis of classified object primitives.

The algorithm starts with the initial image units and proceeds to a pairwise merging of image objects at each iteration. Each image unit (i.e. pixel in 2D or, voxel in our 3D application) is initially considered as an individual image object. The merging criterion is a measure of the local heterogeneity of image objects. This criterion is not a binary "merge" or "not merge" criterion but it evaluates each possible merge with the goal of minimizing the incorporated heterogeneity. The overall heterogeneity is defined as a function of the feature space as a whole, the object size, and the weight assigned to each feature dimension. The algorithm terminates when there are no other pairs of objects satisfying the merging criterion according to the scale factor, which controls the object heterogeneity and subsequently the object size. The higher the value of the scale factor, the higher the internal heterogeneity allowed, which increases the number of the initial image objects (image units) per final image object. In order to assure that the segmentation process is reproducible and to avoid using seed points, the so-called local mutual best fitting heuristics are used (see Wu et al. 2015). Local mutual best fitting assures that each merge is the best possible in the local vicinity of any image object.

By means of FNEA a hierarchical network of image objects can be built in which higher resolution image objects are sub-objects of coarser image objects. This semantic network represents the information of the dataset at different scales (degrees of homogenization) simultaneously and each image object "knows" its content, its neighborhood and its sub-objects (Baatz \& Schape, 1999, 2000).

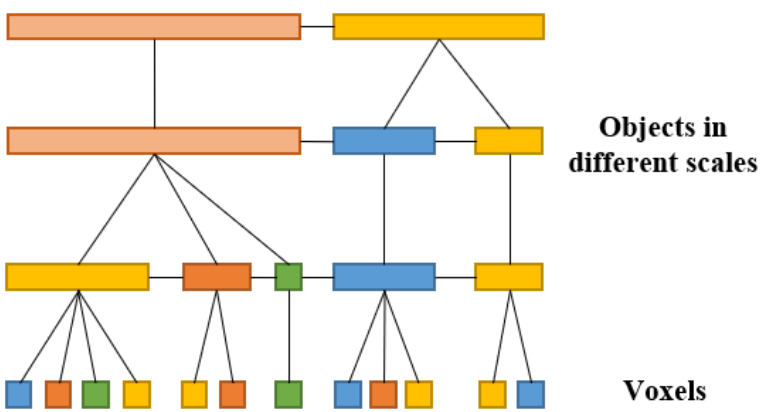

Figure 2. Semantic object network built by FNEA (modified after Baatz and Schape 1999)

\subsection{Morphometric local descriptors}

In this work, the authors utilize geometrical information only; however, reflectance, colour and full-waveform features may also be considered if available. The point cloud does not contain any sort of 3D geometry, and as a result, relations between the points within each voxel can be calculated. A Principal Components Analysis (PCA) is performed for the set of points within each voxel. The voxel size must include at least 4 points within each voxel since this is the minimum number required for performing a PCA, and the corresponding voxel size technically represents the highest achievable resolution of the generated 3D image. PCA allows for encoding the XYZ point set into the so- 
called 3D structure tensor $(3 \times 3$ covariance matrix $)$ and calculates its eigenvalues $\left(\lambda_{1}, \lambda_{2}, \lambda_{3}\right)$ and eigenvectors. Besides the estimation of the orientation through the commonly used normal vector, which is represented by the eigenvector corresponding to the lowest eigenvalue, the estimation of eight additional lowlevel descriptors is facilitated by exploiting the eigenvalues through PCA (Table 1). Moreover, additional 3D properties such as the height difference, local point density and verticality can also be derived for each voxel (Weinmann et al., 2017). These low-level descriptors have also recently become available within the popular open-source 3D point cloud processing software named CloudCompare v2.10.

Additional 3D low-level local descriptors can be provided by Fast Point Feature Histograms (FPFH), as they can approximate the geometry within a voxel with only a few values. FPFH encode the geometrical properties of a volume by generalizing its mean curvature using a multi-dimensional histogram of values providing a discriminating signature for the feature representation. An additional advantage of FPFH is that it copes very well with different point densities (Rusu et al., 2009).

Table 1. The local 3D shape features that can be derived by exploiting the eigenvalues within each voxel

\begin{tabular}{|l|l|}
\hline 3D shape feature & Formulae \\
\hline Linearity & $\mathrm{L}_{\lambda}=\frac{\lambda_{1}-\lambda_{2}}{\lambda_{1}}$ \\
\hline Planarity & $\mathrm{P}_{\lambda}=\frac{\lambda_{2}-\lambda_{3}}{\lambda_{1}}$ \\
\hline Sphericity & $\mathrm{S}_{\lambda}=\frac{\lambda_{3}}{\lambda_{1}}$ \\
\hline Omnivariance & $\mathrm{O}_{\lambda}=\sqrt[3]{\prod_{j=1}^{3} \lambda_{j}}$ \\
\hline Anisotropy & $\mathrm{A}_{\lambda}=\frac{\lambda_{1}-\lambda_{3}}{\lambda_{1}}$ \\
\hline Eigenentropy & $\mathrm{E}_{\lambda}=-\sum_{j=1}^{3} \lambda_{j} \ln \left(\lambda_{j}\right)$ \\
\hline Sum of eigenvalues & $\Sigma_{\lambda}=\sum_{j=1}^{3} \lambda_{j}$ \\
\hline Local surface variation & $\mathrm{C}_{\lambda}=\frac{\lambda_{3}}{\lambda_{1}+\lambda_{2}+\lambda_{3}}$ \\
\hline
\end{tabular}

However, in the current study, a measure of the overall heterogeneity based on both the local dimensionality and the orientation of the scene by means of Equation 1 is proposed.

$$
\mathrm{DoH}=h_{\text {dim }} w_{\text {dim }}+h_{\text {orient }} w_{\text {orient }}
$$

where: $\mathrm{DoH}$ (degree of homogenization) expresses the overall heterogeneity of the forming objects,

$h_{(\text {dim, orient })}$ is the heterogeneity in the dimensionality and orientation, respectively, and

$w_{\text {(dim, orient) }}$ is the weight of the dimensionality and orientation heterogeneity, respectively, in the overall heterogeneity.

\section{APPLICATION}

The proposed 3D point cloud segmentation strategy was applied to a TLS-derived point cloud collected at a landslide-active rock slope, part of the White Canyon, in Interior British Columbia, Canada $\left(50.266261^{\circ},-121.538943^{\circ}\right)$. The White Canyon has been extensively monitored by Queen's University as part of the Canadian Railway Ground Hazard Research Program (RGHRP) since April 2012. A number of different landslide mechanisms have been observed including rockfalls (Kromer et al. 2015), debris flows (Bonneau and Hutchinson 2019), and topples (Rowe et al. 2018) at the study site. Moreover, slope failure processes have created a variable landscape that ranges from debris channels to overhanging blocks and spires of varying lithology. In a number of selected locations, rock sheds have been constructed to protect the track against the aforementioned geohazards. The complex slope geometry (Figure 3) highlights the importance of being able to conduct the morphological slope characterization in $3 \mathrm{D}$

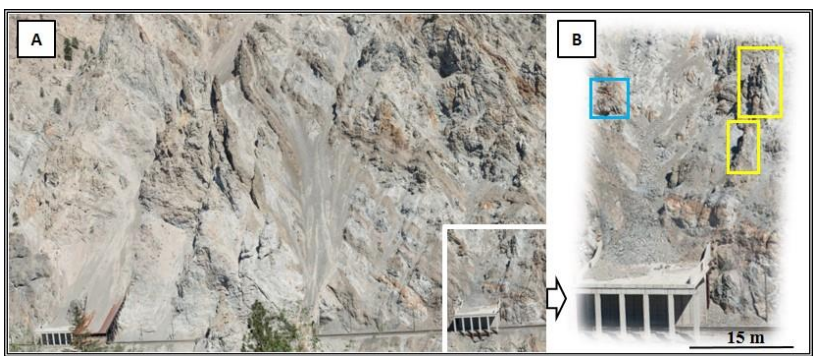

Figure 3. Part of the White Canyon. A) photo showing the overall complexity of slopes in the White Canyon, and B) the study area with spires (yellow frame) and overhanging blocks (light blue frame)

\subsection{Data acquisition and pre-processing}

In order to achieve the best possible 3D representation of the landscape and minimize occlusions, multiple vantage points were selected for the TLS setup. The multiple scans were registered via known stable structure targets and with a rough coarse alignment followed by the implementation of Iterative Closest Point (ICP) algorithm in RiScan Pro with a standard deviation of $1.4 \mathrm{~cm}$. Details regarding the survey plan and pre-processing of the White Canyon datasets can be found in Bonneau et al. (2019). The unevenly distributed point density within the TLS point clouds was resolved by applying a space-based resampling algorithm resulting in an evenly spaced point distribution of approximately $8 \mathrm{~cm}$.

\subsection{Segmentation}

For the segmentation process, both the dimensionality and orientation were calculated and assigned to each voxel, in a 50$50 \%$ weighting, in order to describe the local morphology. Following the work of Brodu and Lague (2012), the dimensionality was expressed by means of normalized eigenvalues $\left(\mathrm{p}_{1}, \mathrm{p}_{2}, \mathrm{p}_{3}\right)$. The orientation was expressed using the slope and aspect of each voxel. These five low-level descriptors technically define the feature space that was used as input to the proposed segmentation/homogenization algorithm. Expressing the content of the scene by means of these two main categories (dimensionality and orientation) should result in objects that better outline and characterize the different structures and geomaterials observed in the scene. In particular, the orientation category is relevant, since strong rock areas can retain higher angles than weathered rock or talus accumulations.

Before the segmentation process begins, the resolution of the 3D image needs to be defined by means of the voxel size. The voxel size is directly related to the point cloud resolution and needs to be carefully adjusted to the purposes of each application based on the user's interpretation. In the presented case, given the available point cloud resolution (approximately $8 \mathrm{~cm}$ ) and the dimensions of the objects of interest, the authors selected a $0.5 \mathrm{~m}$ voxel size so that sufficient geometric information is present within each voxel. A voxel size optimization procedure was conducted for a detailed analytical estimation of the voxel size. Similar to the optimal neighbourhoods in Weinmann et al. (2015), the voxel size was determined by minimizing the XYZ Shannon entropy. In the analysis, a range of $0.5 \pm 0.3 \mathrm{~m}$ was 

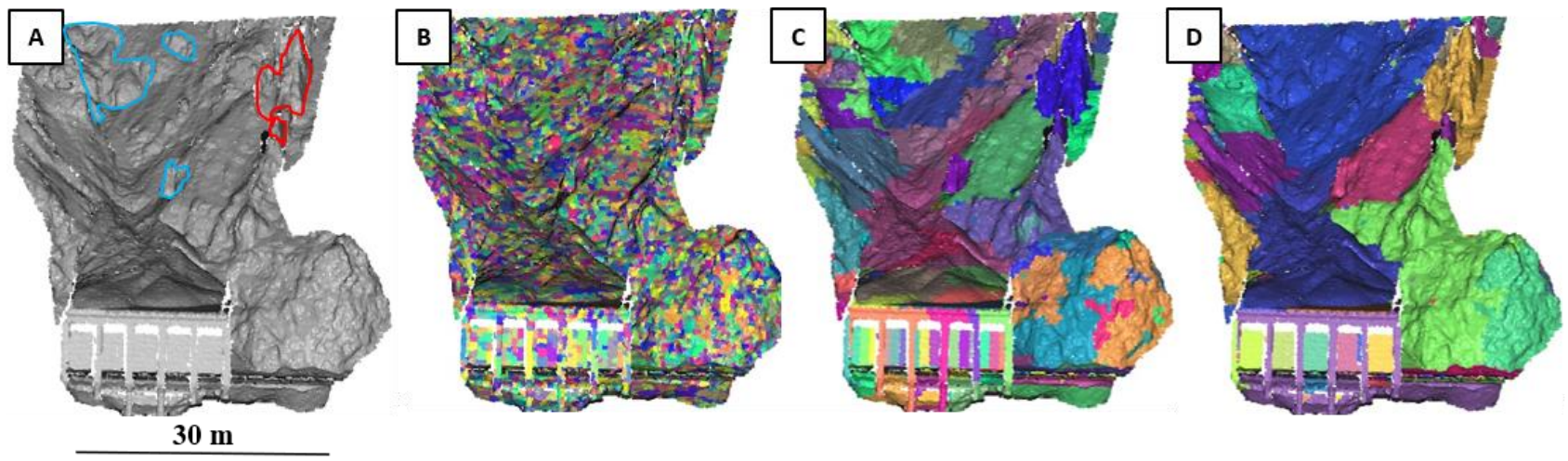

Scale Factor (SF)

Over-segmentation

Under-segmentation

5

16

40

Figure 4. Over- and under-segmentation example for the same voxel size. A) 3D point cloud of the study area with the spires and rock exposures within the main channel enclosed by red and cyan line, respectively, B) segmentation result with $\mathrm{SF}=5, \mathrm{C}$ ) segmentation result with $\mathrm{SF}=16$, and $\mathrm{D}$ ) segmentation result with $\mathrm{SF}=40$

examined with a $1 \mathrm{~cm}$ interval. The minimum average Shannon entropy was achieved at a voxel size of $0.4 \mathrm{~m}$ which was selected to be the resolution of the dataset.

The scale factor (SF) was set to 16 , after an optimization process described in Section 4.3, in order to prevent possible over- or under-segmentation effects as shown in Figure 4. Scale Factor optimization comprises a critical step in the process considering that possible over- and under-segmentation results will dramatically effect the subsequent classification tasks. In Figure 4 , it can be seen that a $\mathrm{SF}=40$ under-segments the scene (Figure 4D) resulting in objects that merge the spires with other neighboring areas and also misses rock exposures within the main channel in the middle of the slope. In contrast, a $\mathrm{SF}=5$ oversegments the scene (Figure 4B) resulting in a large number of almost similar objects within the whole scene, which is something that does not aid subsequent classification.

The objects that are generated can either represent the entire objects of interest themselves or parts thereof. Therefore, when a suitable scale factor is defined the classification of those objects is enhanced by the availability of additional higher-level descriptors such as: the unique outline, the content of each object, and the ability to calculate statistics on either the voxel level or the previous hierarchical levels. In contrast, in voxel- and pointbased classification approaches such additional descriptors are not available due to the unique voxel shape and the pre-defined neighborhood size, respectively, as an effect of the lack of semantic meaning. In this work, when the hierarchical level of a scale factor equals 16, the generated objects comprising the spires (dark blue object in Figure 4C), for instance, are of different size, shape, height and potentially many other additional contextual properties compared to the non-spire objects. With the same logic, the analysis can be performed targeting other structures that may be discerned at lower or higher hierarchical levels (scales).

\subsection{Multi-scale objects evaluation}

The authors considered all the sub-object levels starting from $\mathrm{SF}=1$, up to a scale factor equals $40(\mathrm{SF}=40)$, for the evaluation of the inter-relationships between objects generated at different hierarchical levels and the selection of the optimal SF value. The strategy used for the scale factor optimization was to plot each scale factor against the local variance of the objects comprising the scene at the given hierarchical level (Figure 5), as recommended by Drăguţ et al. (2010). In detail, the point cloud is initially divided into 8810 voxels representing the $3 \mathrm{D}$ space and it is observed that, after the third level $(\mathrm{SF}=3)$ and as the scale factor keeps increasing, the local variance (LV) sharply rises up to 46 , representing the homogenization domain of the curve. The scale factor that corresponds to the peak of the $\mathrm{LV}$ curve $(\mathrm{SF}=16)$ defines the level at which the highest homogenization of the scene is achieved and the objects can be semantically separated more easily. After that point, the LV gradually decreases until the end of the process. This second section of the curve represents the domain where the semantic information starts fading and nonsimilar objects are merged. At the same time, the number of objects rapidly decreases within the homogenization domain. The decreasing number of objects, approximately halved for each level, translates to a gradually increasing number of merges as the segmentation proceeds to higher hierarchical levels. This implies that, for a given scale factor, less possible merges in the scene are found to satisfy the merge criterion, as the overall heterogeneity increases.

For clarity, only four of the forty hierarchical levels were chosen for the graphical demonstration of the semantic "knowledge" that each object has. As shown in Figure 6, starting from level 1 (A) to level 4 (D) and passing though different scales (scale factors), each final object (i.e. the one in the zoomed area) "knows" all the sub-objects that were used to build it in previous levels. It is obvious that the exact same areas include different and decreasing numbers of objects as it advances from lower to higher hierarchical levels.

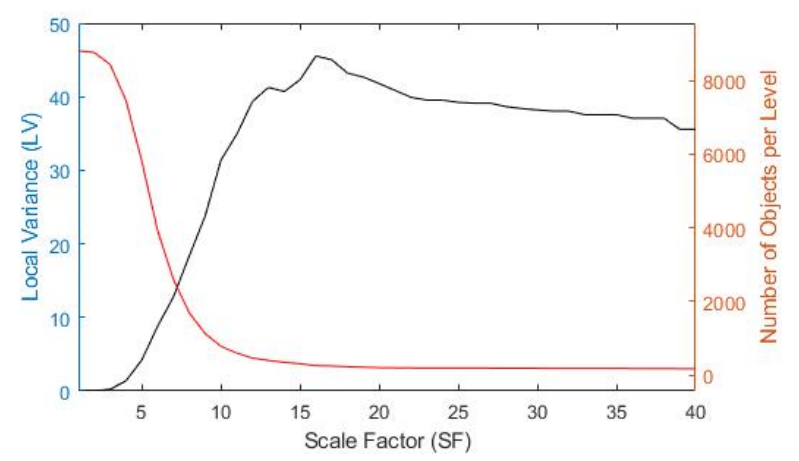

Figure 5. Number of objects generated per hierarchical level (red line) and overall local variance (LV) per hierarchical level (black line) 


\section{DISCUSSION AND CONCLUSIONS}

Object-oriented classification approaches have already become a subject of research in $3 \mathrm{D}$ point cloud semantic labelling (Rutzinger et al. 2008; Mayr et al. 2017). Although recent studies have shown a great potential in engineering geology applications in complex terrains, the final classification result is still limited to single-scale analysis. It relies mainly on the segmentation strategies which usually make use of seeded region growing techniques where the scale of the objects is dependent on the choice of seed points and/or growing thresholds.

The authors have presented a novel point cloud segmentation strategy for object-oriented rock slope evaluation in complex natural environments which makes use of supervoxels. The present approach is a step toward the goal of automated semantic labelling of natural terrain, which is more complex and therefore more challenging than urban terrain, and comprises the first application of the FNEA in the 3D domain using solely geometric information. What makes the natural - and especially the landslide-active - terrains more challenging is the fact that rock structures very rarely represent ideal shape primitives, and generally the colour is not a usable discriminator except where contrast results from different formations, the products of rock weathering or the presence of vegetation. The ongoing research shows the potential to analyse different scales at the same time, which is a main issue in any natural environment application where different structures, even of the same material, appear in different scales simultaneously. In contrast to existing

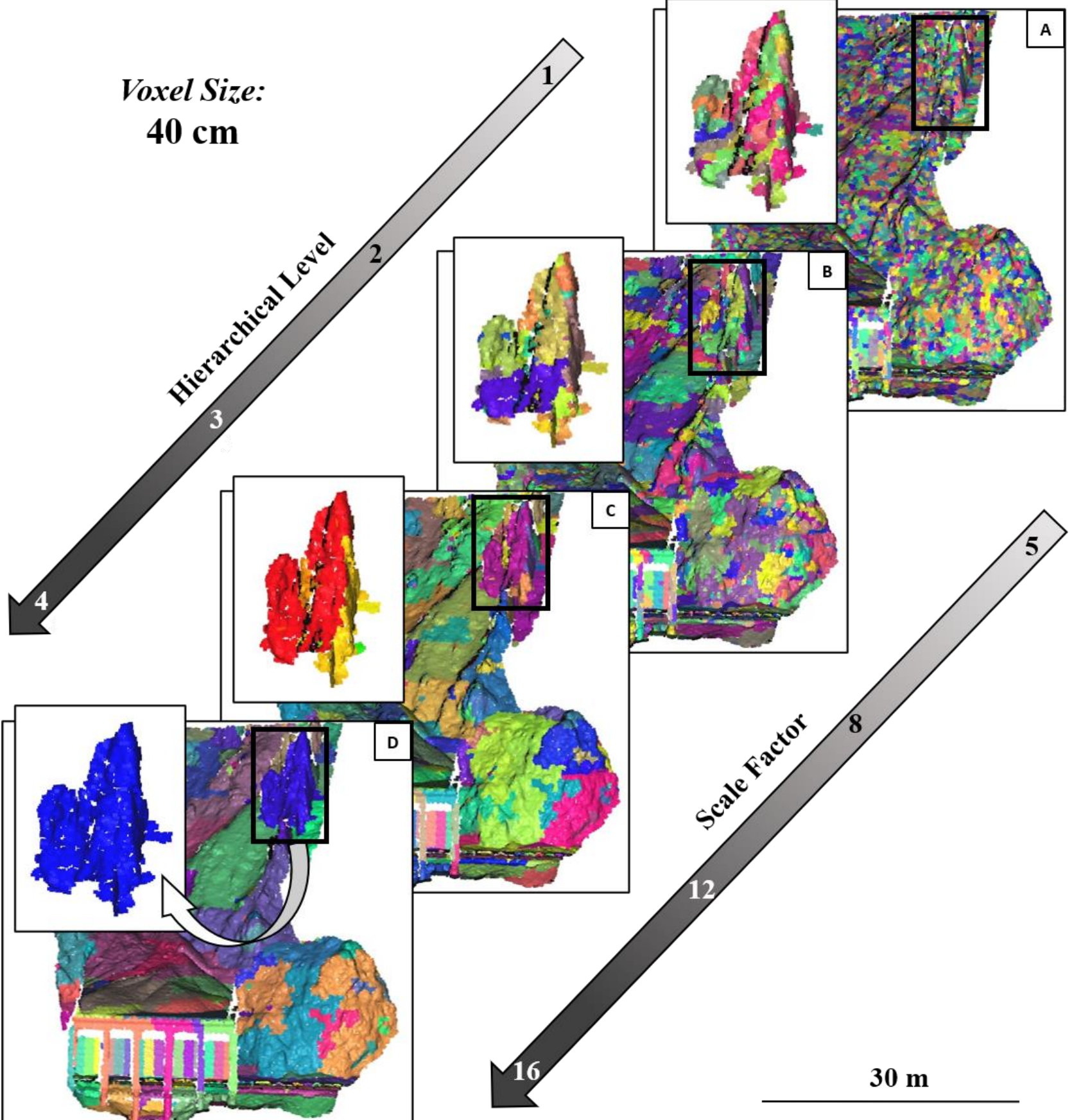

Figure 6. Hierarchical network built by means of FNEA. Four levels were selected from A to D representing scale factors 5, 8, 12, and 16, respectively. Relationships between each level's object and their sub-objects are demonstrated in the zoomed areas 
approaches, the approach works on a voxelized point cloud, using the FNEA and exclusively geometric features to help the supervoxels outline better the real-world objects of interest. The advantages of working in a voxel grid instead of projected image plane (2D) are considerable. One of the most important advantages is the ability to analyse point clouds obtained from different sensor observations. Since the observations are expected to have significant overlap, it is more efficient to segment the overall voxelized point cloud than the individual projected planes (2D observations) (Papon et al., 2013). Moreover, in engineering geological applications the structures of interest and the identification of the critical elements of a rock slope require the fully $3 \mathrm{D}$ representation of the landscape. $2.5 \mathrm{D}$ representations cannot capture overhanging features which are key elements to consider in rockfall hazard assessments since in many cases they do not show any deformation prior to failure (Rowe et al. 2018). Such susceptible rock slope elements typically show substantial variation in volume across a single slope. Thus, the use of such a segmentation strategy with the object size freely adaptable to fit the scale of task is very useful. The proposed strategy builds a hierarchical network of object levels at different scales, simultaneously, storing all the semantic information that is ignored in other $3 \mathrm{D}$ point cloud segmentation strategies. Usually, segmentation techniques are expected to directly and automatically identify all the objects of interest within a scene. This approach, though, only leads to the development of highly specified segmentation strategies applicable only to a limited group of applications (i.e. modelbased). The proposed multi-scale strategy in the current paper examines the local geometric and morphometric information at the level of detail made possible by the point cloud resolution and the voxel size. Subsequently, it starts merging "homogenous" areas maintaining the semantic information associated with each generated object. In that way, one can investigate how the information propagates through the different scales and generate multi-scale higher-level descriptors for the classification of the same area. In addition, the classification of different objects of interest can be conducted at different levels, without overlapping, depending on the scale which is best suited to their identification. However, it is clear that the final result and the whole multi-scale analysis is strongly dependent on the values of the scale factor. The definition of the most suitable scale factor for specific applications is a challenging task and systematic research is required for establishing ways to estimate the most suitable value. In the example application presented in this paper, an optimization strategy based on the local variance was used for the definition of the scale factor value. The methodology appears to perform satisfactorily for detecting the rock spires and homogenizing the scene by general means, however, other scale factor values within the homogenization domain of the local variance curve may be more suitable for the definition of specific object classes.

In any engineering geological mapping practice, either in situ or virtual, visual assessment is the basic tool. Visual assessment was therefore conducted here for the evaluation of the segmentation result since there is no available benchmark repository of similar structures classified. According to Baatz and Schape (1999) $4 \mathrm{pp}$, "as segmentation procedures are used for automation, they are replacing the activity of visual digitizing. No segmentation result - even if quantitatively proofed - will convince, if it does not satisfy the human eye". Visual observation skills to discern rock slope morphology and instability potential are not easily shared among different practitioners, but an automated mapping framework is. Finally, sufficient amounts of training data, as well as well-established classification models should now be used considering all the appropriate object-based morphometric descriptors for the automatic engineering geological labelling of $3 \mathrm{D}$ point clouds of active rock slopes.

\section{ACKNOWLEDGEMENTS}

This research was supported by the Canadian Railway Ground Hazard Research Program (CN Rail, CP Rail, Transport Canada, Geological Survey of Canada) which is funded through a Natural Sciences and Engineering Research Council of Canada (NSERC) CRD grant and the RMC Green Team and the Canadian Department of National Defence.

\section{REFERENCES}

Abellán, A., Oppikofer, T., Jaboyedoff, M., Rosser, N. J., Lim, M., \& Lato, M. J. (2014). Terrestrial laser scanning of rock slope instabilities. Earth Surface Processes and Landforms, $39(1)$

https://doi.org/10.1002/esp.3493

Aijazi, A. K., Checchin, P., \& Trassoudaine, L. (2013). Segmentation based classification of 3D urban point clouds: A super-voxel based approach with evaluation. Remote Sensing, 5(4), 1624-1650. https://doi.org/10.3390/rs5041624

Baatz, M., \& Schape, A. (1999). Object-Oriented and MultiScale Image Analysis in Semantic Networks. 2nd International Symposium: Operationalization of Remote Sensing, 16-20.

Baatz, M., \& Schape, A. (2000). Multiresolution segmentation: An optimization approach for high quality multi-scale image segmentation. In T. Strobl, T. Blaschke, \& G. Griesebner (Eds.), Angewandte Geographische Informations - Verabreitung XII (pp. 12-23). Karlsruhe, Germany. https://doi.org/10.1207/s15326888chc1304_3

Blaschke, T. (2010a). Object based image analysis for remote sensing. ISPRS Journal of Photogrammetry and Remote Sensing, 65(1), 2-16. https://doi.org/10.1016/j.isprsjprs.2009.06.004

Blaschke, T. (2010b). Object based image analysis for remote sensing. ISPRS Journal of Photogrammetry and Remote Sensing, 65(1), 2-16. https://doi.org/10.1016/j.isprsjprs.2009.06.004

Blaschke, Thomas, Hay, G. J., Kelly, M., Lang, S., Hofmann, P., Addink, E., ... Tiede, D. (2014). Geographic ObjectBased Image Analysis - Towards a new paradigm. ISPRS Journal of Photogrammetry and Remote Sensing, 87(January), https://doi.org/10.1016/j.isprsjprs.2013.09.014

Blaschke, Thomas, \& Tomljenović, I. (2012). LiDARscapes and OBIA. American Society for Photogrammetry and Remote Sensing Annual Conference 2012, ASPRS 2012, $44-50$.

Bonneau, D. A., \& Hutchinson, D. J. (2019). The use of terrestrial laser scanning for the characterization of a cliff-talus system in the Thompson River Valley, British Columbia, Canada. Geomorphology, 327, 598-609. https://doi.org/10.1016/j.geomorph.2018.11.022

Bonneau, D. A., Jean Hutchinson, D., DiFrancesco, P. M., Coombs, M., \& Sala, Z. (2019). Three-dimensional rockfall shape back analysis: Methods and implications. Natural Hazards and Earth System Sciences, 19(12), 2745-2765. https://doi.org/10.5194/nhess-19-2745-2019 
Brodu, N., \& Lague, D. (2012). 3D terrestrial lidar data classification of complex natural scenes using a multiscale dimensionality criterion: Applications in geomorphology. ISPRS Journal of Photogrammetry and Remote Sensing, 68(1), 121-134. https://doi.org/10.1016/j.isprsjprs.2012.01.006

Drăguţ, L., Tiede, D., \& Levick, S. R. (2010). ESP: A tool to estimate scale parameter for multiresolution image segmentation of remotely sensed data. International Journal of Geographical Information Science, 24(6), 859871. https://doi.org/10.1080/13658810903174803

Feizizadeh, B., \& Blaschke, T. (2013). A semi-automated object based image analysis approach for landslide delineation. The 2013 European Space Agency Living Planet Symposium, (June 2014), 9-13. https://doi.org/10.1016/j.geomorph.2006.09.023

Hossain, M. D., \& Chen, D. (2019). Segmentation for ObjectBased Image Analysis (OBIA): A review of algorithms and challenges from remote sensing perspective. ISPRS Journal of Photogrammetry and Remote Sensing, 150(February), $115-134$ https://doi.org/10.1016/j.isprsjprs.2019.02.009

Jaboyedoff, M., Oppikofer, T., Abellán, A., Derron, M. H., Loye, A., Metzger, R., \& Pedrazzini, A. (2012). Use of LIDAR in landslide investigations: A review. Natural Hazards, 61(1), 5-28. https://doi.org/10.1007/s11069-010-9634-2

Karantanellis, E., Marinos, V., \& Vassilakis, E. (2019). 3D hazard analysis and object-based characterization of landslide motion mechanism using uav imagery. International Archives of the Photogrammetry, Remote Sensing and Spatial Information Sciences - ISPRS Archives, 42(2/W13), 425-430. https://doi.org/10.5194/isprs-archives-XLII-2-W13-4252019

Kromer, R., Hutchinson, D.J., Lato, M., Gauthier, D., and Edwards, T. 2015. Identifying rock slope failure precursors using LiDAR for transportation corridor hazard management. Engineering Geology, 195, 93-103. doi:10.1016/j.enggeo.2015.05.012

Lato, M., Diederichs, M.S., Hutchinson, D.J., and Harrap, R. 2012. Evaluating roadside rockmasses for rockfall hazards from LiDAR data: Optimizing data collection and processing protocols. Natural Hazards, 60, 831-864. doi: 10.1007/s11069-011-9872-y.

Lato M., Hutchinson D.J., Gauthier D., Edwards T., Ondercin M. 2015. Comparison of ALS, TLS and terrestrial photogrammetry for mapping differential slope change in mountainous terrain. Canadian Geotechnical Journal, 52 (2): 129-140, DOI 10.1139/cgj-2014-0051.

Li, M. (2018). A super voxel-based Riemannian graph for multi scale segmentation of LiDAR point clouds. ISPRS Annals of the Photogrammetry, Remote Sensing and Spatial Information Sciences, 4(3), 135-141. https://doi.org/10.5194/isprs-annals-IV-3-135-2018

Mayr, A., Rutzinger, M., Bremer, M., Oude Elberink, S., Stumpf, F., \& Geitner, C. (2017). Object-based classification of terrestrial laser scanning point clouds for landslide monitoring. Photogrammetric Record. https://doi.org/10.1111/phor.12215

Papon, J., Abramov, A., Schoeler, M., \& Worgotter, F. (2013). Voxel cloud connectivity segmentation - Supervoxels for point clouds. Proceedings of the IEEE Computer Society Conference on Computer Vision and Pattern Recognition, 2027-2034. https://doi.org/10.1109/CVPR.2013.264

Rowe, E., Hutchinson, D.J., and Kromer, R. 2018. An Analysis of Failure Mechanism Constraints on Pre-Failure Rock Block Deformation using TLS and Roto-Translation Methods. Landslides, 15 (3), 409-421. https://doi.org/10.1007/s10346-017-0886-8

Rusu, R. B., Blodow, N., \& Beetz, M. (2009). Fast Point Feature Histograms (FPFH) for 3D registration. In IEEE Int Conf Robot Automation (ICRA) (pp. 3212-3217). https://doi.org/10.1109/robot.2009.5152473

Rutzinger, M., Höfle, B., Hollaus, M., \& Pfeifer, N. (2008). Object-based point cloud analysis of full-waveform airborne laser scanning data for urban vegetation classification. Sensors, 8(8), 4505-4528. https://doi.org/10.3390/s8084505

Sithole, G., \& Vosselman, G. (2003). Automatic structure detection in a point-cloud of an urban landscape. 2nd GRSS/ISPRS Joint Workshop on Remote Sensing and Data Fusion over Urban Areas, URBAN 2003, (May 2014), https://doi.org/10.1109/DFUA.2003.1219959

Telling, J., Lyda, A., Hartzell, P., \& Glennie, C. (2017). Review of Earth science research using terrestrial laser scanning. Earth-Science Reviews, 169(March), 35-68. https://doi.org/10.1016/j.earscirev.2017.04.007

Weinmann, M., Jutzi, B., Hinz, S., \& Mallet, C. (2015). Semantic point cloud interpretation based on optimal neighborhoods, relevant features and efficient classifiers. ISPRS Journal of Photogrammetry and Remote Sensing, 105 , 286-304. https://doi.org/10.1016/j.isprsjprs.2015.01.016

Weinmann, M., Weinmann, M., Mallet, C., \& Brédif, M. (2017). A classification-segmentation framework for the detection of individual trees in dense MMS point cloud data acquired in urban areas. Remote Sensing, 9(3), 0-28. https://doi.org/10.3390/rs903277

Wu, L., Wang, Y., Long, J., \& Liu, Z. (2015). Image and Graphics. (Y.-J. Zhang, Ed.), Lecture Notes in Computer Science (including subseries Lecture Notes in Artificial Intelligence and Lecture Notes in Bioinformatics) (Vol. 9217). Cham: Springer International Publishing https://doi.org/10.1007/978-3-319-21978-3

Ye, S., Pontius, R. G., \& Rakshit, R. (2018). A review of accuracy assessment for object-based image analysis: From per-pixel to per-polygon approaches. ISPRS Journal of Photogrammetry and Remote Sensing, 141, 137-147. https://doi.org/10.1016/j.isprsjprs.2018.04.002 